\title{
Progression of cognitive impairment in stroke/TIA patients over 3 years
}

\author{
Perminder S Sachdev, ${ }^{1,2,3}$ Darren M Lipnicki, ${ }^{1,3}$ John D Crawford, ${ }^{1}$ Wei Wen, ${ }^{1,3}$ \\ Henry Brodaty $1,2,4$
}

- Additional material is published online only. To view please visit the journal online (http://dx.doi.org/10.1136/ jnnp-2013-306776).

${ }^{1}$ Centre for Healthy Brain Ageing, School of Psychiatry, University of New South Wales, Sydney, Australia

2Primary Dementia

Collaborative Research Centre, University of New South Wales, Sydney, Australia ${ }^{3}$ Neuropsychiatric Institute, Prince of Wales Hospital, Sydney, Australia

${ }^{4}$ Academic Department for Old Age Psychiatry, Prince of Wales Hospital, Sydney, Australia

\section{Correspondence to} Professor P Sachdev, Neuropsychiatric Institute, Prince of Wales Hospital, Barker Street, Randwick, NSW 2031, Australia; p.sachdev@unsw.edu.au

Received 12 September 2013 Revised 12 January 2014 Accepted 26 February 2014 Published Online First 21 March 2014

\author{
ABSTRACT \\ Objectives To examine how cognitive deficits progress \\ in the years following a stroke or transient ischaemic \\ attack (TIA).
}

Methods A follow-up study, with neuropsychological and MRI assessments undertaken 3 years after baseline assessments made 3-6 months poststroke in 183 stroke/ TIA patients and 97 healthy controls participating in the Sydney Stroke Study. Additional measures included cardiovascular risk factors and apolipoprotein $\mathrm{E}$ (APOE) genotype.

Results Stroke/TIA patients had poorer cognitive function and more vascular risk factors than controls at baseline, but did not show greater decline in cognitive function over 3 years except for verbal memory. Patients with a subsequent stroke/TIA showed greater decline in global cognitive function and a number of domains. Rates of incident dementia were $5.9 \%$ per year in patients and $0.4 \%$ in controls. Both groups showed increased atrophy of the hippocampus, amygdala and whole brain, and an increase in white matter hyperintensities over 3 years; whole brain atrophy was greater in patients. Cognitive decline was greater in women and in those with smaller hippocampi at baseline. For patients without a subsequent stroke/TIA, those with smaller hippocampi or the APOE \&4 allele had greater global cognitive and verbal memory decline. Conclusions In poststroke patients, cognitive decline was not greater than in comparison subjects, except for verbal memory, unless they had another stroke/TIA.

However, dementia incidence was higher in patients, as might be expected from their poorer baseline cognitive functioning. Smaller hippocampi were associated with an increased risk of decline in memory, and APOE $\varepsilon 4$ was a risk factor in those without a subsequent stroke/TIA.

\section{INTRODUCTION}

Nearly one in four stroke patients has cognitive impairment severe enough to be diagnosed as dementia, ${ }^{1} 2$ and another one in three has milder levels of cognitive impairment. ${ }^{3}$ While some patients may show recovery over an extended period, ${ }^{4}$ an overall decline in cognitive function is to be expected..$^{5-10}$ This is because stroke patients are at increased risk of further cerebrovascular events ${ }^{11}$ and have a heavy burden of often progressive white matter (WM) lesions. ${ }^{12}$ Vascular disease also increases the risk of Alzheimer-type pathology. ${ }^{13}$

Studies examining the progression of cognitive impairment in stroke patients have reported mixed results, largely due to sampling and methodological differences. ${ }^{4-10}$ While earlier studies suggested that mortality rates were higher for vascular dementia $(\mathrm{VaD})$ than Alzheimer's disease (AD) ${ }^{14}$ the rate of cognitive decline in $\mathrm{VaD}$ appears to be slower than AD. ${ }^{15}$ This is supported by the placebo arms of cholinesterase inhibitor trials in VaD. ${ }^{16-18}$ What happens to poststroke patients with only mild impairment over the longer term is less well studied, especially in the absence of further strokes or transient ischaemic attacks (TIAs).

We have previously reported that stroke/TIA patients in the Sydney Stroke Study exhibited mild declines in cognitive functions at 1 year after baseline. ${ }^{19}$ Decline was related to white matter hyperintensities (WMHs) and accentuated if a further cerebrovascular event occurred; education played a protective role. These patients were followed for a further 2 years to examine if a similar pattern of decline continued, and if the predictors of decline showed any change.

\section{METHODS}

Participants

Patients with a recent ischaemic stroke/TIA were recruited consecutively to the Sydney Stroke Study from two teaching hospitals affiliated with the University of New South Wales. Individuals were excluded if diagnosed with dementia or other neurological disorder prior to the stroke/TIA, had severe aphasia limiting assessment or were unable to consent to participate. Healthy controls were unpaid volunteers of similar age and from the same neighbourhood as the patients; they had no history of stroke/TIA, or other neurological or psychiatric disorder. Recruitment occurred over a 38-month period between May 1997 and June 2000. From a total of 1050 patients screened, 252 were considered eligible and recruited. There were also 129 controls recruited.

From all participants initially recruited to the Sydney Stroke Study, the present study included only those with neuropsychological and/or MRI data for one or both of baseline and 3-year follow-up assessments. After excluding five controls reporting a stroke/TIA between baseline and follow-up, neuropsychological data were available for 183 patients and 95 controls. MRI data were available for 117 patients and 79 controls (two controls provided MRI data only). There was more data available at baseline than at follow-up, with the reasons why follow-up data were unavailable including withdrawal from the study for 55 patients (68\%) and 18 controls (78\%), death for 21 patients $(26 \%)$ and three controls (13\%), and geographic 
relocation for five patients (6\%) and two controls (9\%). A smaller number of participants missed the baseline assessment but provided data at follow-up.

For the patients, stroke location was hemispheric in $82 \%$, brainstem in $8 \%$ and cerebellar in $10 \%$, and the NINDS classification was atheroembolic in 51\%, cardioembolic in $19 \%$ and lacunar in $30 \%$.

The study was approved by the ethics committees of the University of New South Wales and the Southeastern Sydney Illawara Area Health Service. All participants provided written informed consent.

\section{Assessments}

\section{Baseline}

Patients undertook functional tests and the Mini Mental State Examination (MMSE) ${ }^{20}$ within 1 week of hospital admission, and a detailed medical and psychiatric examination (including a neuropsychological assessment) 3-6 months after the stroke or TIA. A subgroup also received a brain MRI scan at this time. Controls were assessed upon admission to the study.

\section{Follow-up}

The medical and psychiatric examination (including neuropsychological assessment), functional tests and MMSE were repeated at 12 months and 3 years after baseline. MRI scans were repeated at the 3 -year follow-up only.

\section{Medical, psychiatric and functional assessment}

A medical and psychiatric history was obtained and questionnaires administered, including the Activities of Daily Living $(\mathrm{ADL})^{21}$ and Instrumental ADL (IADL) ${ }^{22}$ scales, and the Social and Occupational Functioning Assessment Scale (SOFAS). ${ }^{23}$ Informants completed the Informant Questionnaire for Cognitive Decline in the Elderly (IQCODE). ${ }^{24}$ The occurrence of an interval stroke or TIA was indicated by history from patient and informant, and a review of the patient's hospital notes.

\section{Neuropsychological assessment}

The test battery addressed these cognitive domains: verbal memory, visual memory, working memory, attention, language, processing speed, visuoconstruction, praxis-gnosis, abstract reasoning, mental flexibility and verbal fluency (online supplementary table S1 shows the tests in each domain). Trained clinical psychologists performed the assessments, which took 2-3 h. Cognitive domain scores were derived by calculating z-scores for each test, using the control group's baseline mean and SD, and averaging across the tests in particular domains. ${ }^{25}$ We calculated a composite neuropsychological test score by averaging the domain scores and transforming the results to z-scores.

\section{MRI scans}

MRI was performed using a $1.5 \mathrm{~T}$ Signa GE scanner (GE Systems, Milwaukee, USA) and the following protocol: $1.5 \mathrm{~mm}$ thick T1-weighted contiguous coronal sections through whole brain using a FSPGR sequence and 3D acquisition (TR $14.3 \mathrm{~ms}$, TE $5.4 \mathrm{~ms}$, FOV $25 \mathrm{~cm}$, inplane resolution $0.977 \times 0.977 \mathrm{~mm}^{2}$ ); and $4 \mathrm{~mm}$ thick (no gap between the slices) T2-weighted FLAIR coronal slices through whole brain (TR $8900 \mathrm{~ms}$, TE $145 \mathrm{~ms}$, TI $2200 \mathrm{~ms}$, FOV $25 \mathrm{~cm}$, matrix size $256 \times 192)$. Scans were processed using Analyze (Mayo Foundation, Rochester Michigan, USA) and SPM99 (Cognitive Neuroscience Group, National Hospital for Nervous Diseases, London). Grey matter (GM),
WM and cerebrospinal fluid (CSF) volumes were obtained after segmentation of T1-weighted images. Total brain volume (TBV) was calculated as GM+WM, intracranial volume (ICV) as TBV $+\mathrm{CSF}$ and brain atrophy as (ICV-TBV)/ICV. Total WMH volume was determined from FLAIR sequences, with WMHs detected and delineated using a computer algorithm designed inhouse and implemented in Matlab. Hippocampal volumes were traced manually by trained operators. The hippocampus was traced until the slice anterior to a clear separation of the crus of the fornix from the wall of the lateral ventricle. The posterior segment of the hippocampus tail was traced from coroniallyoriented images in an anterior-posterior direction until the faciolar gyrus becomes the subsplenium gyrus curving around the postero-inferior margin of the splenium.

\section{Homocysteine and genotyping}

Total homocysteine levels were measured from fasting blood samples using Abbot IMX-automated, fluorescence-based enzyme immunoassays with a range of measurement $0.5-$ $50 \mu \mathrm{mol} / \mathrm{L}$ and demonstrated high repeatability. ${ }^{26}$ Genotyping for polymorphism on the apolipoprotein $\mathrm{E}$ gene (APOE) and the C677T mutation of the methylenetetrahydrofolate reductase enzyme gene (MTHFR) was carried out by PCR-based methods. Participants were classified as $\varepsilon 4$ positive or negative for the APOE4 polymorphism, and as $\mathrm{T}$ positive (TT, TC) or negative (CC) for the MTHFR C677T single nucleotide polymorphism (rs1801133).

\section{Consensus diagnoses}

Consensus diagnoses of dementia were made by a panel comprising a neuropsychiatrist, psychogeriatrician and neurologist, and one or more research psychologists. The criteria for dementia was two or more cognitive domains (not necessarily memory) with impairment (performance $<5$ th centile on at least one test) and evidence of functional decline caused by the cognitive deficits (decline in SOFAS score of $\geq 20$ from the premorbid estimate, or failure on one ADL item or two IADL items due to cognitive deficits).

\section{Statistical analysis}

Demographic and clinical characteristics of patients and controls were compared using $\mathrm{t}$ or $\chi^{2}$ tests. Repeated-measures analyses of neuropsychological and MRI variables were conducted using linear mixed models. Unlike other repeated-measures approaches, linear mixed models are able to include participants missing data for some time-points. ${ }^{27} 28$ The models included group, time and group $\times$ time interaction terms. Neuropsychological analyses featured three groups: patients without an interval stroke/TIA, patients with an interval stroke/TIA and controls. With smaller numbers, MRI analyses featured only two groups, patients and controls. Adjustments for neuropsychological analyses were baseline age and education (both mean-centred) and sex, and for MRI analyses age, sex and time-varying ICV (mean-centred at both assessments). Missing values for education (seven cases) and age (one case) were replaced by zero (as these variables were meancentred). We also used linear mixed models to investigate if certain baseline factors or an interval stroke/TIA predicted declines in composite neuropsychological test score and verbal memory in patients (adjusted for age, sex, education and ICV where appropriate). All linear mixed models had unstructured covariance matrices. Investigations were conducted to determine if and how the presence of any outliers affected our results (outlying values were identified for patients and controls separately as three or more SDs 
Table 1 Demographic and baseline and 3-year follow-up clinical characteristics of patients and controls*

\begin{tabular}{lllc}
\hline & Patients $(\mathrm{n}=183) \dagger$ & Controls $(\mathrm{n}=97) \dagger$ & $\mathrm{p}$ Value \\
\hline Age (years) & $71.91 \pm 9.07$ & $71.07 \pm 6.07$ & 0.36 \\
Females & $74(40.4)$ & $49(50.5)$ & 0.10 \\
Education (years) & $10.10 \pm 2.66$ & $11.75 \pm 3.31$ & $<0.001$ \\
Baseline clinical test scores & & & \\
$\quad$ & $27.83 \pm 2.56$ & $28.78 \pm 1.30$ & $<0.001$ \\
MMSE & $5.26 \pm 1.35$ & $5.97 \pm 0.18$ & $<0.001$ \\
ADL & $6.82 \pm 1.93$ & $7.99 \pm 0.10$ & $<0.001$ \\
IADL & $3.10 \pm 0.25$ & $3.08 \pm 0.16$ & 0.46 \\
IQCODE & $27.60 \pm 3.04$ & $29.19 \pm 0.90$ & $<0.001$ \\
Follow-up clinical test scores & & $6.00 \pm 0.00$ & $<0.001$ \\
$\quad$ MMSE & $5.01 \pm 1.64$ & $7.89 \pm 0.42$ & $<0.001$ \\
ADL & $6.28 \pm 2.31$ & $3.06 \pm 0.26$ & 0.001 \\
IADL & $3.29 \pm 0.60$ & $13(14.0)$ & 0.002 \\
IQCODE & $55(31.4)$ & $33(35.5)$ & $<0.001$ \\
Coronary artery disease & $107(59.4)$ & $7(7.6)$ & 0.04 \\
Hypertension & $29(16.2)$ & $2(2.2)$ & $<0.001$ \\
Diabetes & $44(24.9)$ & $21(24.7)$ & 0.01 \\
Atrial fibrillation & $68(40.2)$ & $40(43.5)$ & 0.004 \\
Hypercholesterolaemia & $108(62.8)$ & $6(6.7)$ & 0.85 \\
Smoker (past or current) & $11(6.1)$ & & \\
Alcohol abuse & $11)$ &
\end{tabular}

${ }^{*}$ Data are presented as either mean + SD or $n(\%)$

tMaximum $n$, with percentages for participants with valid data.

$A D L$, Activities of Daily Living; IADL, Instrumental Activities of Daily Living; IQCODE,

Informant Questionnaire for Cognitive Decline in the Elderly; MMSE, Mini Mental

State Examination.

above or below the mean). Analyses were conducted using IBM SPSS Statistics V.20, and $\mathrm{p}<0.05$ considered statistically significant.

\section{RESULTS}

Demographic and clinical characteristics

Table 1 shows the demographic and clinical characteristics of patients and controls, and the results of comparisons between these. The average age of all participants was 71.6 years (range 49-86), and 43.9\% were women (this did not differ significantly between patients and controls). Controls averaged nearly 12 years of education, which was more than 1.5 years greater than patients. Controls had higher ADL (baseline 5.97 vs 5.26), IADL (baseline 7.99 vs 6.82) and MMSE (baseline 28.78 vs 27.83) scores than patients; they also had lower IQCODE scores at follow-up, but not at baseline (see table 1 for details). Compared with controls, the MMSE, ADL and IADL scores of patients decreased significantly from baseline to follow-up $(p=0.012, p=0.005$ and $p<0.001$, respectively). Patients were also more likely than controls to have history of coronary artery disease (31\% vs 14\%), hypertension (59\% vs 36\%), diabetes ( $16 \%$ vs $8 \%)$, atrial fibrillation $(25 \%$ vs $2 \%)$ and hypercholesterolaemia ( $40 \%$ vs $25 \%$ ), and to be past or current smokers $(63 \%$ vs 44\%). Patients missing follow-up data did not differ from patients with follow-up data on sex, education or baseline IQCODE, but were older (74.56 vs 70.05 years, $\mathrm{p}=0.001)$ and had lower baseline ADL (4.79 vs 5.60, p<0.001), IADL $(5.92$ vs $7.48, \mathrm{p}<0.001)$ and MMSE $(27.11$ vs $28.40, \mathrm{p}=0.002)$ scores. There were no significant differences in the rates of cardiovascular risk factors (see online supplementary table S2 for details), WMH volume or brain atrophy, but patients missing follow-up data had smaller amygdalae and hippocampi than patients with follow-up data $(1.47 \pm 0.28 \mathrm{~mL}$ vs $1.65 \pm 0.30 \mathrm{~mL}$, $\mathrm{p}=0.014$ and $5.28 \pm 0.76 \mathrm{~mL}$ vs $5.61 \pm 0.76 \mathrm{~mL}, \mathrm{p}=0.056$, respectively).

\section{Interval strokes and TIAs}

In all, 42 patients $(23.0 \%)$ had at least one stroke or TIA between baseline and follow-up, including 15 cases of TIA only, five of stroke only, 18 of both TIA and stroke, and four unknown. Patients with an interval stroke/TIA did not differ significantly from those without an interval stroke/TIA on age, education or APOE4 status, but experienced greater declines in MMSE and composite neuropsychological test scores $(p=0.021$ and $\mathrm{p}<0.001$, respectively).

\section{Cognitive functioning}

The numbers of participants with data available for the neuropsychological analyses are shown in table 2. Table 3 shows estimated mean values from the fitted linear mixed models for the controls and two patient groups (with and without an interval stroke/TIA). Across the cognitive domains investigated, scores were typically lower for patients than controls at both baseline and follow-up. For patients with an interval stoke/TIA, follow-up scores were frequently lower than baseline scores. The extent to which these differences are statistically significant is indicated by the parameter estimates also shown in table 3, and which are for the interactions between time and patient group (vs controls), separately for each group of patients. A negative parameter estimate indicates a greater decline in patients than controls, and a positive parameter estimate indicates a smaller decline in patients than controls. Patients with an interval stroke/TIA exhibited significantly greater decline in performance than controls on six of the 12 measures, whereas patients without an interval stroke/TIA declined more rapidly than controls only on verbal memory. For almost every cognitive domain, there were one or two participants for whom the difference between baseline and follow-up was an outlying value. Excluding these made no difference to the results, except for

Table 2 Numbers of participants contributing to the linear mixed models analyses of neuropsychological and MRI data

\begin{tabular}{|c|c|c|c|c|c|}
\hline \multirow[b]{2}{*}{ Assessment periods } & \multicolumn{3}{|l|}{ Neuropsychological } & \multicolumn{2}{|l|}{ MRI } \\
\hline & Patients with interval stroke/TIA & Patients without interval stroke/TIA & Controls & All patients & Controls \\
\hline Baseline only & 64 & 17 & 22 & 49 & 13 \\
\hline Follow-up only & 9 & 4 & 0 & 16 & 5 \\
\hline Baseline and follow-up & 68 & 21 & 73 & 52 & 61 \\
\hline Total baseline & 132 & 38 & 95 & 101 & 74 \\
\hline Total follow-up & 77 & 25 & 73 & 68 & 66 \\
\hline
\end{tabular}

TIA, transient ischaemic attack. 
Table 3 Cognitive domain scores at baseline and follow-up in controls and patients either with or without an interval stroke/TIA and interactions (each patient group vs controls $\times$ time)*

\begin{tabular}{|c|c|c|c|c|c|c|c|c|c|c|}
\hline \multirow[b]{3}{*}{ Domain } & \multicolumn{2}{|l|}{ Controls } & \multicolumn{4}{|c|}{ Patients with interval stroke/TIA } & \multicolumn{4}{|c|}{ Patients without interval stroke/TIA } \\
\hline & \multirow[b]{2}{*}{ Baseline } & \multirow[b]{2}{*}{ Follow-up } & \multirow[b]{2}{*}{ Baseline } & \multirow[b]{2}{*}{ Follow-up } & \multicolumn{2}{|l|}{ Interaction } & \multirow[b]{2}{*}{ Baseline } & \multirow[b]{2}{*}{ Follow-up } & \multicolumn{2}{|l|}{ Interaction } \\
\hline & & & & & Parameter estimate & p Value & & & Parameter estimate & p Value \\
\hline Attention & $-0.11 \pm 0.13$ & $-0.19 \pm 0.14$ & $-0.51 \pm 0.19$ & $-1.19 \pm 0.23$ & $-0.60 \pm 0.25$ & 0.01 & $-0.83 \pm 0.11$ & $-0.73 \pm 0.13$ & $0.17 \pm 0.17$ & 0.32 \\
\hline Verbal memory & $-0.10 \pm 0.10$ & $0.16 \pm 0.13$ & $-0.08 \pm 0.16$ & $-0.27 \pm 0.21$ & $-0.44 \pm 0.21$ & 0.03 & $-0.12 \pm 0.09$ & $-0.41 \pm 0.12$ & $-0.54 \pm 0.14$ & $<0.001$ \\
\hline Visual memory & $-0.07 \pm 0.11$ & $0.31 \pm 0.12$ & $-1.00 \pm 0.17$ & $-1.04 \pm 0.20$ & $-0.41 \pm 0.21$ & 0.06 & $-0.86 \pm 0.10$ & $-0.48 \pm 0.12$ & $0.00 \pm 0.15$ & 0.98 \\
\hline Processing speed & $-0.10 \pm 0.16$ & $-0.02 \pm 0.17$ & $-1.05 \pm 0.25$ & $-1.70 \pm 0.29$ & $-0.72 \pm 0.23$ & 0.002 & $-1.09 \pm 0.15$ & $-1.14 \pm 0.17$ & $-0.12 \pm 0.16$ & 0.44 \\
\hline Reasoning & $-0.11 \pm 0.12$ & $0.06 \pm 0.13$ & $-0.99 \pm 0.19$ & $-0.86 \pm 0.21$ & $-0.04 \pm 0.22$ & 0.84 & $-1.11 \pm 0.11$ & $-0.73 \pm 0.12$ & $0.21 \pm 0.15$ & 0.16 \\
\hline Working memory & $-0.10 \pm 0.10$ & $-0.17 \pm 0.11$ & $-0.61 \pm 0.15$ & $-1.01 \pm 0.18$ & $-0.33 \pm 0.16$ & 0.03 & $-0.66 \pm 0.09$ & $-0.69 \pm 0.10$ & $0.04 \pm 0.11$ & 0.70 \\
\hline Language & $-0.01 \pm 0.15$ & $0.16 \pm 0.17$ & $-0.33 \pm 0.22$ & $-1.33 \pm 0.28$ & $-1.16 \pm 0.27$ & $<0.001$ & $-0.82 \pm 0.13$ & $-0.83 \pm 0.16$ & $-0.17 \pm 0.18$ & 0.36 \\
\hline Praxis-gnosis & $-0.08 \pm 0.18$ & $0.03 \pm 0.14$ & $-0.92 \pm 0.28$ & $-0.57 \pm 0.24$ & $0.23 \pm 0.36$ & 0.53 & $-0.92 \pm 0.16$ & $-0.47 \pm 0.14$ & $0.32 \pm 0.25$ & 0.20 \\
\hline Visuoconstruction & $-0.11 \pm 0.13$ & $-0.14 \pm 0.14$ & $-1.16 \pm 0.21$ & $-1.50 \pm 0.24$ & $-0.30 \pm 0.22$ & 0.18 & $-0.76 \pm 0.11$ & $-0.87 \pm 0.13$ & $-0.6 \pm 0.15$ & 0.66 \\
\hline Mental flexibility & $-0.10 \pm 0.27$ & $-0.19 \pm 0.22$ & $-1.27 \pm 0.45$ & $-2.14 \pm 0.42$ & $-0.78 \pm 0.50$ & 0.11 & $-2.14 \pm 0.25$ & $-1.64 \pm 0.23$ & $0.59 \pm 0.32$ & 0.06 \\
\hline Verbal fluency & $-0.10 \pm 0.10$ & $-0.11 \pm 0.12$ & $-0.85 \pm 0.15$ & $-1.16 \pm 0.20$ & $-0.29 \pm 0.20$ & 0.14 & $-0.89 \pm 0.09$ & $-0.85 \pm 0.12$ & $0.04 \pm 0.14$ & 0.74 \\
\hline Composite score & $-0.10 \pm 0.09$ & $-0.03 \pm 0.10$ & $-0.75 \pm 0.14$ & $-1.22 \pm 0.15$ & $-0.54 \pm 0.10$ & $<0.001$ & $-0.96 \pm 0.08$ & $-0.84 \pm 0.08$ & $0.04 \pm 0.07$ & 0.56 \\
\hline
\end{tabular}

the additional finding of a significant decline in verbal fluency for patients with an interval stroke/TIA.

MRI

The numbers of participants with data available for the MRI analyses are shown in table 2 . The estimated mean values (figure 1 ) and parameter estimates (table 4) of fitted models indicate significantly greater WMH volumes in patients (baseline $37 \mathrm{~mL}$; follow-up $42 \mathrm{~mL}$ ) than controls (baseline $16 \mathrm{~mL}$; follow-up $21 \mathrm{~mL}$ ). Hippocampus and amygdala volumes decreased significantly and WMH volume and brain atrophy increased significantly over time across all participants. The increase in brain atrophy of around 0.1 across all participants was significantly (though only slightly) greater in patients than controls.

\section{Incident dementia}

The rate of incident dementia between baseline and follow-up was significantly higher in patients (20 from $113,17.7 \%)$ than controls (one from 85, 1.2\%; $\chi^{2}=13.97, p<0.001$ ). This analysis of incident dementia rates excluded 32 patients with dementia at baseline and participants lost to the 3-year follow-up not diagnosed with dementia at 12 months (18 controls, 59 patients). To see if these exclusions could have influenced our results, we conducted sensitivity analyses with all
Figure 1 Change in brain atrophy and hippocampus, amygdala and total white matter hyperintensity (WMH) volumes between baseline and follow-up in stroke patients (black circles) and controls (open circles). Points represent the estimated mean values $( \pm \mathrm{SE})$ for the group (patients, controls) $\times$ time (baseline, follow-up) interaction term of linear mixed models. Atrophy was calculated as the ratio of cerebrospinal fluid (CSF) to intracranial volume (ICV).
Hippocampus

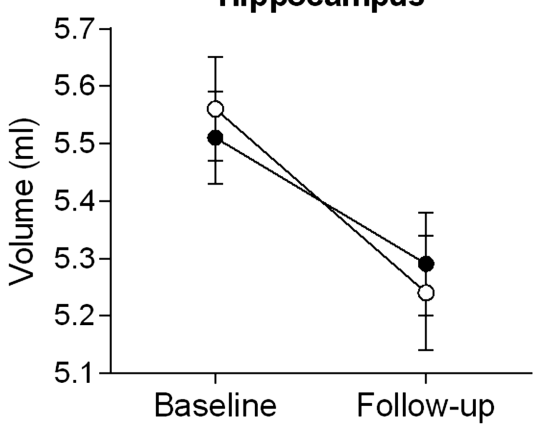

WMH

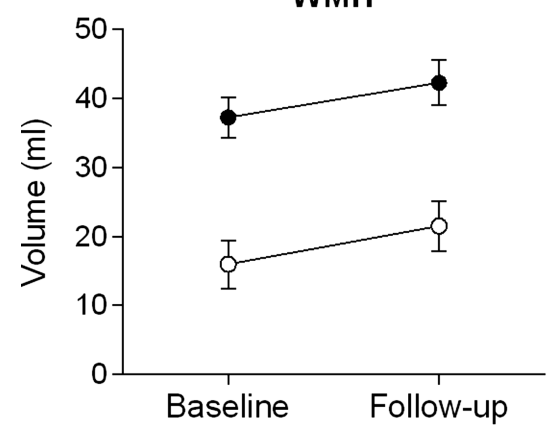

Amygdala

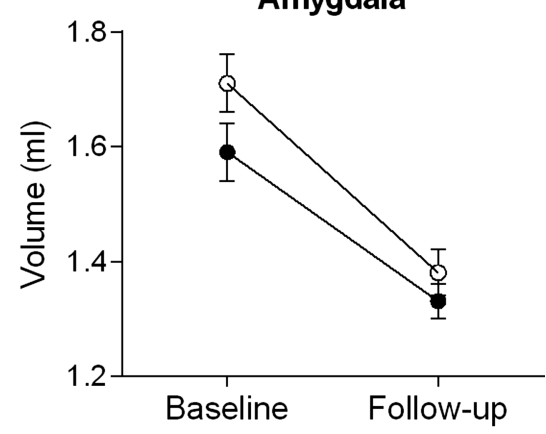

Atrophy

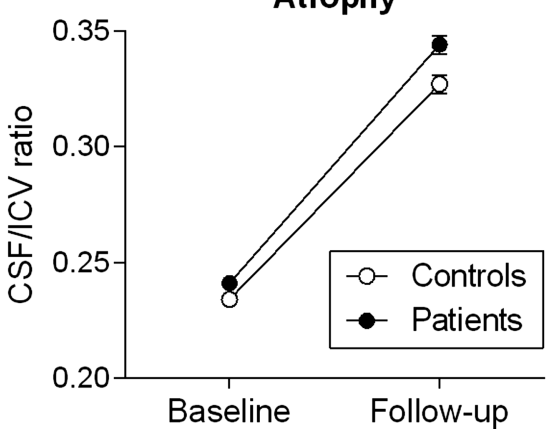


Table 4 Linear mixed model parameter estimates $( \pm$ SE) for MRI variables

\begin{tabular}{|c|c|c|c|c|c|c|}
\hline \multirow[b]{2}{*}{ MRI variable } & \multicolumn{2}{|c|}{ Patients vs controls } & \multicolumn{2}{|c|}{ Follow-up vs baseline } & \multicolumn{2}{|c|}{$\begin{array}{l}\text { Change over time, patients vs } \\
\text { controls }\end{array}$} \\
\hline & Estimate & $\mathrm{p}$ Value & Estimate & $\mathrm{p}$ Value & Estimate & $p$ Value \\
\hline Hippocampus* & $-0.04 \pm 0.11$ & 0.67 & $-0.31 \pm 0.11$ & 0.007 & $0.09 \pm 0.15$ & 0.55 \\
\hline Amygdala* & $-0.12 \pm 0.06$ & 0.06 & $-0.33 \pm 0.05$ & $<0.001$ & $0.07 \pm 0.08$ & 0.34 \\
\hline WMHs* & $0.02 \pm 0.00$ & $<0.001$ & $0.00 \pm 0.00$ & $<0.001$ & $0.00 \pm 0.00$ & 0.71 \\
\hline Atrophy† & $0.00 \pm 0.00$ & 0.10 & $0.09 \pm 0.00$ & $<0.001$ & $0.10 \pm 0.00$ & 0.04 \\
\hline
\end{tabular}

*Estimates calculated using volume in $\mathrm{mL}$ and adjusted for age, sex and time-varying intracranial volume.

tEstimates adjusted for age and sex.

WMHs, white matter hyperintensities.

missing participants classified as either developing or not developing incident dementia. The results were unchanged in these sensitivity analyses, with patients continuing to show a higher rate of incident dementia than controls $(\mathrm{p} \leq 0.001)$.

\section{Predictors of cognitive decline in stroke patients}

Investigations of whether certain baseline factors predicted declines in composite neuropsychological test score and verbal memory were performed for all stroke patients, and repeated for those without an interval stroke/TIA. Table 5 shows parameter estimates from the fitted linear mixed models for the interactions between predictor and time (follow-up vs baseline), adjusted for age, sex and education. A negative parameter estimate indicates greater decline and a positive parameter estimate indicates less decline if meeting a categorical factor (eg, female vs male) or for increasing values of a continuous factor (eg, hippocampus volume). Across all patients, there were greater declines in composite score for women and individuals with smaller hippocampi. Patients without an interval stroke/TIA showed greater declines in composite score if $A P O E \varepsilon 4$ positive. The same baseline factors had some associations with verbal memory decline. Across all patients, women declined more than men (estimate: $-0.51, \mathrm{SE}=0.19, \mathrm{p}=0.011$ ). Higher $\mathrm{WMH}$ volume was also associated with greater verbal memory decline when an additional four patients with high WMH volumes $(>3 \mathrm{SD}$ above the mean) were included (estimate: $-7.28, \mathrm{SE}=3.14, \mathrm{p}=0.024$; otherwise estimate: $-2.82, \mathrm{SE}=4.94, \mathrm{p}=0.571$ ). Patients without an interval stroke/TIA showed significantly greater verbal memory decline if $A P O E \varepsilon 4$ positive (estimate: $-0.95, \mathrm{SE}=0.35$, $\mathrm{p}=0.011)$, and tended to show greater decline if having smaller hippocampi (estimate: $0.522, \mathrm{SE}=0.26, \mathrm{p}=0.055)$. An interval stroke/TIA was associated with greater decline in composite score (estimate: $-0.58, \mathrm{SE}=0.12, \mathrm{p}<0.001$ ), but not in verbal memory (estimate: $0.10, \mathrm{SE}=0.23, \mathrm{p}=0.647$ ).

Table 5 Linear mixed model parameter estimates $( \pm \mathrm{SE})$ for baseline predictors of decline in composite neuropsychological test scores in stroke/ TIA patients*

\begin{tabular}{|c|c|c|c|c|}
\hline \multirow[b]{2}{*}{ Predictor } & \multicolumn{2}{|l|}{ All patients } & \multicolumn{2}{|l|}{ No interval stroke/TIA } \\
\hline & Parameter estimate & p Value & Parameter estimate & $\mathrm{p}$ Value \\
\hline \multicolumn{5}{|l|}{ Demographic/clinical } \\
\hline Age in years & $0.00 \pm 0.00$ & 0.82 & $0.00 \pm 0.00$ & 0.67 \\
\hline Female & $-0.31 \pm 0.11$ & 0.01 & $-0.22 \pm 0.10$ & 0.04 \\
\hline Education in years & $0.02 \pm 0.02$ & 0.20 & $0.01 \pm 0.01$ & 0.59 \\
\hline MMSE score & $-0.02 \pm 0.03$ & 0.48 & $-0.04 \pm 0.03$ & 0.20 \\
\hline IQCODE score & $-0.43 \pm 0.30$ & 0.15 & $0.14 \pm 0.28$ & 0.61 \\
\hline \multicolumn{5}{|l|}{ Cardiovascular risks } \\
\hline Hypertension & $-0.16 \pm 0.12$ & 0.20 & $-0.12 \pm 0.11$ & 0.25 \\
\hline Diabetes & $0.01 \pm 0.16$ & 0.90 & $0.04 \pm 0.15$ & 0.80 \\
\hline High cholesterol & $0.16 \pm 0.12$ & 0.18 & $-0.06 \pm 0.10$ & 0.56 \\
\hline Smoker & $0.21 \pm 0.12$ & 0.09 & $0.17 \pm 0.11$ & 0.12 \\
\hline \multicolumn{5}{|l|}{ Laboratory measures } \\
\hline Homocysteine in $\mu \mathrm{mmol} / \mathrm{L}$ & $0.00 \pm 0.00$ & 0.89 & $0.00 \pm 0.00$ & 0.85 \\
\hline APOE4 \&4 positive & $0.19 \pm 0.21$ & 0.36 & $-0.48 \pm 0.17$ & 0.009 \\
\hline MTHFR T positive & $-0.12 \pm 0.20$ & 0.53 & $0.14 \pm 0.18$ & 0.43 \\
\hline \multicolumn{5}{|l|}{ MRI } \\
\hline Atrophy & $-0.33 \pm 2.79$ & 0.90 & $-1.51 \pm 2.15$ & 0.48 \\
\hline Hippocampus in $\mathrm{mL} t$ & $0.36 \pm 0.13$ & 0.01 & $0.30 \pm 0.11$ & 0.008 \\
\hline WMHs in L & $-0.93 \pm 2.55$ & 0.71 & $-1.05 \pm 2.44$ & 0.66 \\
\hline
\end{tabular}




\section{DISCUSSION}

This study reports the progression of cognitive impairment in stroke/TIA patients enrolled in the Sydney Stroke Study over 3 years. Patients did not typically decline more than controls in most cognitive domains, but they started from a lower baseline. The exception was that patients showed greater verbal memory decline $(-0.45 \mathrm{SD})$ than controls. This difference was large and highly significant, but in being for only one cognitive domain contrasts with the far more pervasive decline observed in the $23 \%$ of patients with an interval stroke/TIA. These patients showed significantly greater declines in MMSE and composite cognitive scores, as well as on scores for verbal, visual and working memory, language, attention, and processing speed. These findings expand upon our 1-year follow-up report for this cohort, ${ }^{19}$ when patients similarly showed greater declines in MMSE, composite and verbal memory scores than controls. Patients also showed greater decline than controls in visuoconstruction over 1 year, but not over 3 years. Patients with a subsequent stroke showed accelerated decline in global cognitive functioning over both follow-up intervals. However, our 3-year findings identify a number of individual cognitive domains in which patients with a subsequent stroke show accelerated decline.

The rates of dementia present a somewhat different picture, with $17.7 \%$ of patients diagnosed with dementia in this period; an annual incidence of $5.9 \%$. Only $1.2 \%$ of controls received this diagnosis. The apparent discrepancy between the somewhat similar rates of decline in cognitive domain scores and the higher dementia incidence in patients may be explained by patients having lower baseline cognitive performance.

It is important however to consider participant attrition, especially for patients. The majority of attrition was due to death or ill-health, and those not completing the 3-year follow-up were 5 years older and had lower baseline MMSE scores (-2.9 points). This indicates selective attrition of the more cognitively impaired, and an outcome that is likely to be positively biased. The use of linear mixed models attempted to take this into consideration, and sensitivity analyses showed that dementia rates were higher in patients.

Previous reports have provided varying rates of cognitive decline in stroke patients, with annual declines in MMSE of $-1.1,{ }^{5}-1.77,,^{15}-0.82^{16}$ and $-0.78^{18}$ points reported by different studies. Other studies have also reported low rates of incident dementia in stroke patients. ${ }^{9}{ }^{29-31}$ Our study supports this, and also suggests that, as expected, those with another stroke/TIA show greater decline. An interval stroke/TIA accelerated cognitive change by -1.62 (vs -0.08 ) points in our patients, consistent with previous reports. ${ }^{5} 3031$ The concept of multi-infarct dementia that this finding supports must however be elaborated, as another predictor of decline in those with an interval stroke/TIA was WM lesions, when their level was high. The cognitive impairment, and thereby dementia, is therefore the combined effect of both large and small vessel disease, with the former making a bigger contribution, and an interval stroke/TIA having a more discernible effect is not surprising. A recent review concluded that a stroke doubled the risk of dementia incidence in individuals $>65$-years-old, but had no effect in individuals $>85$-years-old. ${ }^{29}$ The rate of decline, with or without an interval stroke/TIA, was however less than that seen in early $\mathrm{AD} .^{32}$ Interventions trials in $\mathrm{VaD}$ must also take this into consideration when planning the sample size and duration of intervention.

The greater decline in verbal memory we observed is worthy of comment. We have previously argued that frontal-executive dysfunction is an early feature of vascular cognitive disorders, while early decline in memory favours $\mathrm{AD}^{2}$ Deducing the neuropathological substrate of the memory decline is however difficult, given the logical memory subtest of the WMS-R addresses complex processes involving registration, retention and recall of structured information, any of which may be affected by WM lesions or front-subcortical system disturbances without the hippocampus being directly affected. ${ }^{33}$ The memory decline may therefore occur from vascular or Alzheimer-type pathology, and the cognitive profile does not permit discernment between these.

We examined neuroimaging and genetic factors to investigate the pathological basis of cognitive decline. Patients and controls both showed reductions in whole brain, hippocampal and amygdala volumes and increases in WMH volumes, but whole brain atrophy was greater in patients. We also found that smaller baseline hippocampi predicted greater memory decline. However, the attrition of participants with smaller hippocampi and amygdalae at baseline suggests that associations between these structures and cognitive decline may have been underestimated. Verbal memory decline in patients without an interval stroke/ TIA was also associated with having an APOE $\varepsilon 4$ allele, which is strongly associated with Alzheimer-type pathology but only inconsistently with vascular cognitive disorders. ${ }^{34}$ Previous studies have shown that stroke affects dementia incidence in $A P O E$ \& 4 negative individuals only. ${ }^{35} 36$ Our finding relating $A P O E \varepsilon 4$ to cognitive decline in the absence of stroke is consistent with this. We infer that the cognitive decline in our stroke patients is probably due to a combination of vascular and neurodegenerative processes, with the former predominating in those with repeat vascular events.

Consistent with previous reports, ${ }^{29}$ decline was not explained by baseline cognitive function, prestroke cognitive decline or education. Cerebrovascular risk factors also did not emerge as significant predictors of cognitive change. While hypertension, diabetes, hypoxic/ischaemic and other cerebrovascular risk factors contribute to vascular cognitive disorders, they are usually not identified as predicting decline..$^{5-7} 910$ Some possible explanations for this include: (i) the risk factors do not contribute beyond accounting for the stroke and WMH risk at baseline; (ii) the contribution is accounted for by their impact on baseline cognitive status; or (iii) the small magnitude of decline limits the statistical power to detect risk factors.

Our study is characterised by detailed and careful assessments but has some limitations. First, many potential participants were excluded for being unable to complete assessments, biasing our sample toward the less severely affected. Second, attrition, due mainly to illness or death, biased our sample toward the healthy end of the spectrum. This is inevitable in stroke patients, but we attempted to overcome this limitation by using linear mixed models and performing a sensitivity analysis. Nevertheless, it is possible that the true magnitude of associations between predictors and cognitive decline was attenuated in our study by survivor bias. Third, controls had more years of education than patients, but this was controlled for statistically. Fourth, our study probably underestimated the extent of vascular pathology as aspects of cerebrovascular disease such as silent lacunar infarct and microbleeds were not examined. Fifth, the use of self-report measures in participants with memory problems could be considered a challenge to the validity of some results. Sixth, a follow-up of 3 years may have been insufficient time for vascular risk factors or stroke/TIA to exert the full effects.

In conclusion, our study supports a picture of slow cognitive decline in stroke/TIA patients who do not experience a further 
stroke or TIA. In contrast, patients reporting further strokes or TIAs during follow-up exhibited significant and much greater cognitive decline. This was evident for general cognitive functioning and many cognitive domains, with possible contributions from both vascular and neurodegenerative processes.

Acknowledgements We thank the Prince of Wales and St George Hospital neurologists for permission to study their patients; SEALS Laboratories for assistance in blood collection; Karen Berman, David Gillies, Leslie Howard, Megan Jones, Nicole Kochan, Jeffrey Looi, Eveline Milne, Amy Ross, Penny Sawdy, Jamie Sims and Alessandro Zagami for contributing to the study; and Angie Russell for manuscript preparation.

Contributors Study concept and design: PSS and HB. Acquisition of data: PSS and WW. Analysis and interpretation of data: PSS, DML and JDC. Drafting of the manuscript: PSS and DML. Critical revision of the manuscript for important intellectual content: PSS, DML, JDC, WW and HB. Guarantor: PSS.

Funding This study was supported by grants from the National Health and Medical Research Council of Australia (ID222842), the Rebecca Cooper Foundation, and the Fairfax Family Foundation (PSS and HB).

Competing interests PSS, DML, JDC and WW report no competing interests. HB is an investigator for Pfizer, Novartis, Janssen, Lundbeck and Lilly, a sponsored speaker for Pfizer, Novartis, Janssen and Nutricia, and is on the advisory boards of Pfizer, Novartis, Janssen, Lundbeck and Nutricia.

Ethics approval Ethics committees of the University of New South Wales and the Southeastern Sydney Illawara Area Health Service.

Provenance and peer review Not commissioned; externally peer reviewed.

\section{REFERENCES}

1 Desmond DW, Moroney JT, Sano M, et al. Incidence of dementia after ischemic stroke: results of a longitudinal study. Stroke 2002:33:2254-60.

2 Sachdev PS, Brodaty H, Valenzuela MJ, et al. The neuropsychological profile of vascular cognitive impairment in stroke and TIA patients. Neurology 2004;62:912-19.

3 Sachdev PS, Brodaty H, Valenzuela MJ, et al. Clinical determinants of dementia and mild cognitive impairment following ischaemic stroke: the Sydney Stroke Study. Dement Geriatr Cogn Disord 2006;21:275-83.

4 Desmond DW, Moroney JT, Sano M, et al. Recovery of cognitive function after stroke. Stroke 1996;27:1798-803.

5 Aharon-Peretz J, Daskovski E, Mashiach T, et al. Natural history of dementia associated with lacunar infarctions. J Neurol Sci 2002;203-204:53-5.

6 del Ser T, Barba R, Morin MM, et al. Evolution of cognitive impairment after stroke and risk factors for delayed progression. Stroke 2005;36:2670-5.

7 Nyenhuis DL, Gorelick PB, Freels S, et al. Cognitive and functional decline in African Americans with $\mathrm{VaD}, \mathrm{AD}$, and stroke without dementia. Neurology 2002;58:56-61.

8 Srikanth VK, Anderson JF, Donnan GA, et al. Progressive dementia after first-ever stroke: a community-based follow-up study. Neurology 2004;63:785-92.

9 Tham W, Auchus AP, Thong M, et al. Progression of cognitive impairment after stroke: one year results from a longitudinal study of Singaporean stroke patients. J Neurol Sci 2002;203-204:49-52.

10 Wentzel C, Rockwood K, MacKnight C, et al. Progression of impairment in patients with vascular cognitive impairment without dementia. Neurology 2001;57:714-16.

11 Phillips RA. A review of therapeutic strategies for risk reduction of recurrent stroke. Prog Cardiovasc Dis 2008;50:264-73.

12 Sachdev $\mathrm{P}$, Wen W, Chen $\mathrm{X}$, et al. Progression of white matter hyperintensities in elderly individuals over 3 years. Neurology 2007;68:214-22.
13 Kalaria RN, Akinyemi R, Ihara M. Does vascular pathology contribute to Alzheimer changes? I Neurol Sci 2012;322:141-7.

14 Chui H, Gonthier R. Natural history of vascular dementia. Alzheimer Dis Assoc Disord 1999;13:S124-30.

15 Segal-Gidan FI, Lyness SA, Chui HC. A comparison of mental status decline in ischemic vascular dementia and Alzheimer's disease. Neurology 1999;52:A486-A87.

16 Black S, Roman GC, Geldmacher DS, et al. Efficacy and tolerability of donepezil in vascular dementia: positive results of a 24-week, multicenter, international, randomized, placebo-controlled clinical trial. Stroke 2003;34:2323-30.

17 Erkinjuntti T, Kurz A, Gauthier $\mathrm{S}$, et al. Efficacy of galantamine in probable vascular dementia and Alzheimer's disease combined with cerebrovascular disease: a randomised trial. Lancet 2002:359:1283-90.

18 Wilkinson D, Doody R, Helme R, et al. Donepezil in vascular dementia: a randomized, placebo-controlled study. Neurology 2003:61:479-86.

19 Sachdev PS, Brodaty $\mathrm{H}$, Valenzuela MJ, et al. Progression of cognitive impairment in stroke patients. Neurology 2004;63:1618-23.

20 Folstein MF, Folstein SE, McHugh PR. "Mini-mental state". A practical method for grading the cognitive state of patients for the clinician. J Psychiatr Res 1975;12:189-98

21 Katz S, Ford AB, Moskowitz RW, et al. Studies of illness in the aged. The index of Adl: a standardized measure of biological and psychosocial function. JAMA 1963;185:914-19.

22 Lawton MP, Brody EM. Assessment of older people: self-maintaining and instrumental activities of daily living. Gerontologist 1969;9:179-86.

23 American Psychiatric Association. Diagnostic and Statistical Manual of Mental Disorders. 4th edn. Washington, DC: American Psychiatric Association, 1994.

24 Jorm AF, Jacomb PA. The Informant Questionnaire on Cognitive Decline in the Elderly (IQCODE): socio-demographic correlates, reliability, validity and some norms. Psychol Med 1989;19:1015-22.

25 Massaro JM, D'Agostino RB Sr, Sullivan LM, et al. Managing and analysing data from a large-scale study on Framingham Offspring relating brain structure to cognitive function. Stat Med 2004;23:351-67.

26 Nexo E, Engbaek F, Ueland PM, et al. Evaluation of novel assays in clinical chemistry: quantification of plasma total homocysteine. Clin Chem 2000;46:1150-6.

27 Kliegl R, Wei P, Dambacher M, et al. Experimental effects and individual differences in linear mixed models: estimating the relationship between spatial, object, and attraction effects in visual attention. Front Psychol 2010;1:238.

28 West BT. Analyzing longitudinal data with the linear mixed models procedure in SPSS. Eval Health Prof 2009:32:207-28.

29 Savva GM, Stephan BC. Epidemiological studies of the effect of stroke on incident dementia: a systematic review. Stroke 2010;41:e41-6.

30 Srikanth VK, Quinn SJ, Donnan GA, et al. Long-term cognitive transitions, rates of cognitive change, and predictors of incident dementia in a population-based first-ever stroke cohort. Stroke 2006;37:2479-83.

31 Tzourio C, Anderson C, Chapman N, et al. Effects of blood pressure lowering with perindopril and indapamide therapy on dementia and cognitive decline in patients with cerebrovascular disease. Arch Intern Med 2003;163:1069-75.

32 Mayeux R, Small SA, Tang M, et al. Memory performance in healthy elderly without Alzheimer's disease: effects of time and apolipoprotein-E. Neurobiol Aging 2001;22:683-9.

33 Budson AE, Price BH. Memory dysfunction. N Engl J Med 2005;352:692-9.

34 Rocchi A, Orsucci D, Tognoni $G$, et al. The role of vascular factors in late-onset sporadic Alzheimer's disease. Genetic and molecular aspects. Curr Alzheimer Res 2009:6:224-37.

35 Ivan CS, Seshadri S, Beiser A, et al. Dementia after stroke: the Framingham Study. Stroke 2004:35:1264-8.

36 Zhu L, Fratiglioni L, Guo Z, et al. Incidence of dementia in relation to stroke and the apolipoprotein $E$ epsilon4 allele in the very old. Findings from a population-based longitudinal study. Stroke 2000;31:53-60. 